\title{
Strategies for Integrating Cultural Values in Ethics Education for Nurses
}

\author{
Moliehi Rosemary Mpeli ${ }^{1}$ \\ ${ }^{1}$ School of Nursing, University of the Free State, Bloemfontein, South Africa \\ Correspondence: Moliehi Rosemary Mpeli, School of Nursing 205 Nelson Mandela Avenue, Bloemfontein 9301. \\ South Africa. Tel: 275-1-401-2506. E-mail: mpelirm@ufs.ac.za
}

Received: March 14, 2018 Accepted: May 9, 2018 Online Published: June 11, 2018

doi:10.5539/gjhs.v10n7p61 URL: https://doi.org/10.5539/gjhs.v10n7p61

\begin{abstract}
Background: Limited usage of cultural values has prevailed in ethics education, probably due to controversial issues of cultural relativism. There is, however, an urgent need to bridge the gap between education and practice to ensure cultural appropriate ethical nursing care.

Methods: A literature review was done to identify transformative theory and strategies that may encourage students to recall, reflect and discuss self-identified morality.

Results: The proposed approach draws on transformative learning theory of Mezirow, and introduces strategies for aligning values with ethical theories. The article suggests that instead of imposing moral theories on students, transformative learning strategies should be implemented to reframe students' ingrained values and realise the assimilation of these values in moral theories.

Conclusion: Reframing and assimilation are necessary to bridge the gap between ethics education and practice in nursing. The strategies may enable intercultural dialogues that are deemed necessary for harmonious interaction among people with varied and dynamic cultural values and identities.
\end{abstract}

Keywords: cultural values ethics education, nursing

\section{Introduction}

The need to integrate cultural values within the realm of nursing ethics education has been a focus of interest in recent years. For example, Lutzen (1997) recommends that nursing education should reflect on individual cultural values, while Kuhse and Singer (2009) suggest that individual cultural values should be given prima facie consideration, as well as some adjudication in cases where conflicts arise with the inherent dignity and fundamental rights of others. Despite this noticeable interest, little or limited usage of cultural values has prevailed in ethics education, probably due to different moral codes amongst cultures and possible controversial issues of cultural relativism (Rachels \& Rachels, 2012, p.14). It is essential that a considered position be taken in the realisation and affirmation of diverse cultural values, especially in the global context where training of nurses is defined by multiculturalism and diversity.

Douglas et al. (2011, p. 319) encourage the use of critical reflection in the awareness of culture and cultural values in nursing. Considering the richness of cultural values from an anthropological viewpoint, it is essential to acknowledge that the notions translating from these values may also exhibit both benefits and drawbacks in the practice of nursing care. For example, confidentiality may be undermined in societies where illnesses and their care imply a societal effort, and inclusion and involvement of relatives and friends. It is therefore essential to develop an approach that explores the useful aspects of these values, from the insider perspective, for the benefit of nursing practice. Therefore, appropriate ethical frameworks for enhancement of ethical competency in culturally diverse nursing education contexts are suggested.

It is apparent that the current framework for ethics education is not effective in developing ethical competency, as there has always been some disquiet about the gap between ethics education in nursing and the practice thereof (Esterhuizen 2006; Pattison \& Wainwright, 2010; White, Phakoe, \& Rispel, 2015). One assumption for this ineffectiveness may be the fact that many nursing institutions base the teaching of ethics on traditional moral theories and the international code of ethics for nurses (Geyer, 2015). Although these codes of ethics are founded on respect for human dignity, they failed to close the gap between knowledge and practice. Bertolami (2004, p. 417) 
describes this gap as "a disconnect between knowing what's right and doing it, and understanding the principles of ethics at an intellectual level and applying them in daily life". This disconnect is evident in the majority of nurses who although acquainted with the international codes of ethics for nurses, fail to apply it in practice (White et al., 2015). It is widely believed that the codes of conduct alone cannot support the moral decision-making of practicing nurses and midwives (Esterhuizen 2006; Pattison \& Wainwright, 2010). This belief stems from an understanding that ethics education that relies solely on knowledge transmission of these ethical frameworks cannot make one ethical. The challenge with which nursing ethics education is presented, is therefore the incongruences between the ethics theoretical knowledge and the individual nurse, while diverse cultural values are further widening the gap between ethical knowledge and its implementation within the premise of a diverse population.

Many authors recommend a new approach to ethics education, one that would allow and promote self-reflection. For example, White et al. $(2015$, p. 6$)$ are of the opinion that an approach capable of linking ethical competency with the ability to provide good care would be ideal for ethics education. This approach would indeed be ideal; however, nursing care across cultures must avoid the marginalisation of cultural values, especially in the complex context of diversity. Ludwick and Silva (2000) hold that cultural attitudes and values in nursing may lead to conflict if there are no nursing strategies devised to address them. Likewise, Kikuchi (1996) attests that ethics education should address diversity, as diversity offers unique skills that may carry valuable insights for the improvement of ethics practice. Although cultural diversity in its very essence would include differences in moral perceptions and moral judgements that can be in tension and contention with universal ethical principles or standards (Mele \& Sanchez-Runde, 2013), there is a need for a different educational strategy that would take into account pluralistic values that are relevant for nursing education and practice in a multicultural context.

\section{Methodology}

The aim of this article is therefore to describe an educational approach that may reasonably address the needs of the diverse populations of our global world. Furthermore, it is important to identify and describe some strategies for reflecting on cultural values and experiences towards alignment of these cultural values with the ethical frameworks pertinent to good care by the diverse populations. A literature review was done to identify transformative strategies that may encourage students to recall, reflect and discuss self-identified morality. As the article is focusing on transformative ethical education, the article pays particular attention to Mezirow's (1997) work on transformative learning and transformative education. Given the challenges of cultural relativism and different moral codes that are likely to emerge from diverse cultures, Mezirow's theory of transformative practice and learning (see Mezirow, 1997) was therefore deemed appropriate for this article. For the purpose of applicable strategies, the researcher implemented the experience gained while participating in the Difficult Dialogues project. The project introduced a range of strategies for having civil discourse around topics that are considered controversial. The Difficult Dialogues project was initiated by the University of the Free State (UFS) in response to the diversity challenges that transpire within the institution. The consultant from the University of Alaska Anchorage (UAA), United States of America, was invited to run the project in 2012 and 2013, in collaboration with the UFS. This article therefore, presents a discussion of the application of the transformative theory as an approach to ethics education, and then provides strategies for bringing about transformative learning.

\section{A Transformative Approach to Ethics Education}

The transformative learning approach is related to the general education of an adult learner, and not to issues of cultural diversity. It is extrapolated here because it may benefit the multicultural context of our global world. The discussion around the proposed approach for the ethics education of diverse cultures is therefore based on the transformative learning theory as conceived by Mezirow (1997). Transformative learning is "defined as process of effecting change in frame of reference, that selectively shape and delimit our expectations, perception and feelings" (Mezirow, 1997, p. 5). This frame of reference is believed to impede self-reflection and the discriminative and integrative processing of new ideas. Obstruction occurs because the frame of references are like filters through which we interpret our experiences and set forth our line of action (Mezirow, 1997). Thus, any information that does not fit these perceptions is considered irrelevant, unworthy and weird, and we tend to give it a label, founded on complex feelings, beliefs, judgements and attitudes (Mezirow, 1997).

Accordingly, these frames of reference are likely to have implications for nursing ethics education, as the students oriented towards a certain frame are likely to impose it on others as well as on patients. For example, orientation towards relational autonomy may be a frame that may possibly limit and undermine self-determination demonstrated and valued by other cultural groups of patients. If this is overlooked, the patient's care is likely to be negatively affected, especially in multicultural society of our global world.

According to Mezirow (1997), these frames of reference stem from the cultural assimilation influences of the 
family unit as the primary caregiver. Because the frames of reference are entrenched within the cognitive, conative and emotional component of our experiences, they orientate our habits of feeling and acting (Mezirow, 1997). The frames carry more weight because they constitute a set of codes from our cultural, educational, political, economic and psychological context (Mezirow, 1997). The two frames of reference identified by Mezirow (1997) are habit of mind and viewpoint. The viewpoint is understood to be susceptible to change, because of its accessibility to awareness and the feedback of others, while the habit of mind is change-resistant because it is subjected to a set of codes. It can be argued reasonably that the habit of mind is responsible for the gap between knowing the theory of what is right, and the application of these principles in daily practice (Bertolami, 2004); hence, the identified gap between ethics education and practice in nursing.

Mezirow (1997) contends that, although some frames may be deemed beneficial, without exposure to objective and subjective reframing such frames may never lead to autonomous thinking and critical reflection. To be objectively reframed, this habit of mind should be engaged with discourses that will enable discovery, explanation, interpretation, validation and judgement of a frame, with other individuals as well as with one's own critical reflection (Mezirow, 2003). While subjective reframing requires assessment of one's own ideas and beliefs, it is also crucial for self-reflection and personal transformation (Mezirow, 1997). Thus, the purpose of ethics education must be to initiate this process of identifying, engaging, understanding and interpreting such complex life worlds in collaboration with the individual learners, for the purpose of self-transformation necessary to bridge the gap between ethics education and practice.

Transformative learning encompasses different learning strategies in dealing with the habit of mind, and communicative learning has proved to enable and effect change (Mezirow, 1997). Such learning involves at least two people or more, striving towards understanding, interpreting and justifying a belief and experience. Critical-dialectical discourse, as one method implicated in communicative learning, proves to be valuable in exploring, analysing, judging and synthesising values, beliefs and experiences (Mezirow, 2003). This discourse is coupled with critical reflection, in the sense that the arguments are subjected to analysis and evaluation before being synthesised, while the dialectical aspect involves uncovering frames in relation to everyday activities and underlying social structures (Biazar, 2014).

The standards governing such engagements oblige the participants to be open-minded, empathetic listeners, and to suspend premature judgement, as well as to seek common ground for the purpose of efficient transformation (Mezirow, 2003). These conditions encourage clarification of misconceptions and critical reflection before the frames can change through accretion or attrition (Mezirow, 1997).

The understanding of how these frames operate is essential for planning educational strategies to facilitate transformative education. Transformative education involves redesigning the role of an educator, as the educator needs to identify the classroom conditions necessary to enable a smooth process (Mezirow, 2003). The educator in transformative learning is expected to set the objectives, plan the activities, and to be a facilitator during the discussions, with limited authority on subject matter (Mezirow, 1997). Besides the arrangements for a safe environment, which includes a favourable atmosphere and venue for group activities, the educator should ensure that each student has an opportunity to assume the full range and variety of roles within the process of discourse. These roles include the request to advance a belief or value, challenging a belief, defending and explaining a position, as well as assessing evidence and judging an argument (Mezirow, 1997).

Given the value of critical-dialectical discourse in transformation, there is no mention, however of what should be done if the discourse comes to a dead end, a problem commonly encountered in narrative ethics (Baldwin, 2015). This is what Baldwin (2015, p. 187) calls a narrative foreclosure in which a frame may be 'locked', demonstrating limited possibility for change of a perspective. This challenge may be solved by the educator's expertise and appropriate advanced planning of activities.

The route for changing the frame references may be emotionally charging for those involved and is likely to be strewn with challenges (Sue, Lin, Torino, Capodilupo, \& Rivera 2009). Concerns about moral relativism, for example, as discussed in literature (Rachels \& Rachels, 2012, p.14), are expected to manifest. The historical contexts of countries that are in transition from monoculture to multiculturalism education may be marked by lack of interest, and this may imply a measure of resistance. These challenges can justifiably be dealt with through the use of specific tools that are recognised for promoting respectful transformative discourses on controversial topics and contexts (Landis, 2008, p. i). Classrooms, Landis writes, "should be laboratories where discourse on controversial issues are discussed so as to rebuild a culture of civil discourse, necessary for the reframing of old tenets for the emerging world". 


\section{Strategies for Transformative Learning}

Reframing of the old tenets should be tailored towards a gradual awareness and consciousness. This awareness should promote inclusion, active engagement and participation of the learners. The concepts or objects that are within the context of the learner should therefore be used as sources for discourse (Mezirow, 1997). For this purpose, the two strategies proposed in this article are discussed. For acknowledging diversity, as well as appreciating the worlds that shape us (i.e. class, race and ethnic identities), each student should be required to introduce an object of ancestry, (Landis, 2008) e.g. dancing, chanting, herbs, reeds and food pertinent to care and caring. This strategy is within the premise that no student's background should be marginalised and that all individuals bring value to the civilised exchange (Landis, 2008). This is to be followed by another strategy, one aimed at instilling the habit of doubting, in which one is encouraged to navigate through perspectives that are less appreciated when ethical issues are being dealt with. The rationale behind these strategies is that emotions and feelings are key factors for effective transformation (Dirkx, 2006). It is believed that activation of sensory and imagery cognitive processes can allow construction of new points of view and interconnection with other dimensions of cognition (Lawrence, 2008).

\subsection{The Circle of Objects}

According to Landis (2008, p. 92), "the circle of objects is an exercise that respectfully acknowledges the varieties of cultural heritage and introduces visual and kinesthetic elements into a discussion". For the purpose of ethics, the students should bring to class an object that represents how they see and value themselves within the notion of care. This must be based on a previous and memorable moment of caring for someone. This item may be either an artefact, poem, or a performance of an act, e.g. a drama or dance originating in their background. Their choice of objects should be focused on vulnerable conditions of being human, such as birth, illness, suffering and dying.

The identified objects must be accompanied by a written narrative, for the purpose of the discovery and construction of the self who is at the centre of narrative gravity, (Baldwin, 2015; Walker, 1989). The significance of this narrative lies in its potential to facilitate discussion and to aid analysis, as well as to constitute a portfolio of ethical development. The narrative should be narrated in three steps. Firstly, the learners need to state clearly the reasons and values that inspired the choice of object; second, they have to describe the caring incident related to the object, and, thirdly, they have to conclude with the description of emotions that were felt during and after the caring incident. The essence of each story and the exchange of views during the discussions can be tape-recorded if the group is amenable. If not, the discussions could be handwritten. As these discussions may be intimating, Brookfield \& Preskill (2005) propose that dispositions such as hospitality, participation, mindfulness, humility, mutuality, deliberation, appreciation, hope and self-determination should guide the discussions. Cannaerts, Gastmans, \& Dierckx de Casterle (2014) commend a nonthreatening setting, where significant time for discussion can be allowed. Even though these discussions are expected to yield good results, Sue, Lin, Torino, Capodilupo, \& Rivera (2009) warn that narrative sharing may make the students vulnerable, because of the exposure of their sentiments and beliefs. Ground rules must accordingly be set before engaging in the exercise. The guidelines as proposed by Merculieff \& Roderick (2013) should be followed (see Figure 1).

Preparation: Ask each person to bring into class an object that reflects something about his or her ancestry, cultural heritage, class background or other feature you wish to illuminate. Explain the purpose of the exercise and how it will work. Give students several days to choose their object and consider their response.

Ground rules: treat each other with respect. Everyone has his or her own truth. Listen, be polite, considerate and thoughtful.

Sharing: Arrange the chairs in a circle around a low table. Invite each person, one at a time, to place his or her object on the table and to talk about its links to his or her culture, history, traditions or other topic under discussion.

Tips: Consider speaking first yourself, to model the act of self-disclosure and to demonstrate a time guideline of two or three minutes. After that, let the students speak in whatever order they wish. Honour the silence between speakers.

Figure 1. Guidelines and ground rules for discussions. Adopted from Merculieff \& Roderick (2013)

The facilitator should be first to present and describe his or her object, and thereafter he or she should encourage probing and questioning from the students. This is a form of icebreaker and role modelling for the learning curve, 
which the discussion should follow. The students are then invited to present their objects, which should be made visible to everyone only when being discussed. If it is an artefact, it may be placed on the table. Each student should be given a chance to present his or her story in connection with the object, and this should be followed by probing, questioning and validation from other students, geared towards explanation, interpretation, common understanding and judgement. As a rule, each student should also fulfil the other roles of discourse, as proposed by Mezirow (1997). As Owens (2007) commends, the narratives may be dealt with through classroom criticism, in which probative questions may lead the narrator and the listeners to underlying insights and overarching principles. Again such questioning may encourage the narrator to examine his or her story critically (Owens, 2007).

Once everyone has been given a chance to try all roles, all the verbatim accounts should be transcribed, and the values and habits within the text should be highlighted. These transcripts should be discussed in small groups (five students per group) that should be formed based on homogeneity of values. It is in these homogenous groups that students may feel free to talk about the emotions that accompany their narratives. The size of the group reduces any potential feeling of intimidation.

In these small groups, students should recall the emotions and sentiments that were experienced during the time of an incident or action, and identify whether the same emotions still exist when the story is retold. Further, they should try to make explicit the rationale behind such feelings. The group should appoint a scribe to capture the list of all the emotions. One person should present their group account, and the rest of the members should answer questions from other groups.

It is only after this exercise that the facilitator may analyse the transcripts for strengths and limitations, and compare them with the emotions and feelings presented. At this point, the alignment with current ethical frameworks may be possible, as the results of the analysis would give an indication of the prevailing values. The students may now be introduced to moral theories and codes of ethics. These ethical frameworks ought to be discussed amongst the students to aid interpretation and common understanding. Once there is a common fundamental understanding of the ethical framework, the students are to compare the values and principles enshrined within the ethical frameworks with those values translating from their own narratives. It is here where some of the students' values may overarch with values from ethical frameworks, while others may be different. This activity of comparing one's values with ethical frameworks, may carry an assessment value, and should be an individual effort.

\subsection{The Five-Minute Rule}

The outcomes of the comparison activity should be followed by another planned activity, aimed at reframing and thereby realigning one's own values with the ethical frameworks. For the purpose of reframing, the values that are deemed neglected or unfairly disregarded in the previous activity, should be used to steer emotions. The idea is based on Mezirow's (1997) claim that these frames are attached to emotions and feelings. In addressing this, Brookfield (2002) suggests that students should be given conceptual tools to challenge their own emotions and feelings, as they are an integral part of the meaning-making process that underlies the unconscious forms associated with learning (Dirkx, 2006). In order to allow construction of new points of view and to enable a connection with cognitive dimensions, Brookfield and Preskill (2005) propose a strategy called the five-minute rule. Prior to this activity, the students should go to their previous small groups that were established according to homogeneity of values. Then the facilitator should assign a written document to each group with values, beliefs or ideas that contrast with the group's values and then allow the members of each group to respond to these ideas.

The strategy is aimed at embracing and entertaining, for a short period, ideas, beliefs or values that are considered foreign and/or dubious. The aim is to allow students to heighten their capacity to deal with implausible ideas (Brookfield \& Preskill, 2005). For example, students who embrace individuality with values that include self-determination, may be asked to entertain the value of communitarianism that relies on relational autonomy. While those whose values disregard self-determination should entertain respect for autonomy. Landis (2008, p.109) advises that this activity should be guided by statements such as:

What is interesting or helpful about the view? What are some intriguing features of this view that other might not have noticed? What would be different and what are the implications if you believed this view, or accepted it as true? In what sense and under what conditions might this idea be true?

According to Brookfield and Preskill (2005, p. 134), this simple moment of empathising could "break the dialogical jam" that usually obstructs discussion and the process of mutual understanding. The cracking of this dialogical jam pivots on the fact that those who compose questions are also expected to provide the answers. It is in this reversal of roles in seeking knowledge that students learn to answer their own questioning by challenging their 
capacity to interrogate issues taken for granted. As Brookfield and Preskill (2005, p. 134) affirm, this process helps one to be empathic, the very trait that can transform the experience of care. It is envisaged that this activity will trigger some emotions and feelings, and this is crucial for transformation, as these emotions deepen the process of meaning-making and reflection (Dirkx, 2006).

The responses to the guiding questions should then be written down as a group consensus, and subsequently be presented to the whole class. After presentation, each student should reflect on the activity and describe its influence on his or her emotions. The reflective writing activity may serve as part of the assessment exercise.

The presentation should be followed by another written assignment, in which the students should apply the outcome of this exercise to their original narrative, so as to characterise their narrative from this new point of view. The students should describe the emotions experienced during the activity. The documents of the activities should then be compiled into portfolios, for the purpose of personal development.

\section{Conclusions}

Transformative learning theory offers a promising approach to ethics education for multicultural society, especially in today's world where nursing care is across cultural context. This theory demonstrates a unique means by which a wide spectrum of values can align within a coherent ethical framework. The importance of transformative learning and strategies discussed in this article lies in their ability to induce intercultural dialogues. What is intriguing about this theory is its ability to guide students through the process of self-discovery with others as co-constructors. As Jack Mezirow (1997) suggests, the habits of mind are difficult to change outside the province of awareness. It is through the prejudices and myths that others hold about one's culture that your explanation and defence may result in genuine and authentic awareness of own habits of mind. Consequently, the disconnect between ethics education and ethics in practice may never be resolved if ethics education does not invest in the process of self-discovery for the students.

It must however be noted that this suggested approach to ethics education has not yet been validated empirically. Yet, various studies (Squire, 2016) that have previously implemented these strategies have yielded good results. Although the benefits of these strategies have not been tested within the context of ethics education, the dense description of these strategies in this article invites nurse educators to implement them in contexts that are diverse in cultures, values, beliefs and experiences, to test their usability, strengths and weaknesses. It is therefore recommended that future research be directed towards evaluating the use of the transformative approach in ethics education, as proposed in this article, as well the exploration of the experiences of students and educators regarding the approach.

It can be concluded that the transformative strategies are essential in aligning cultural values with the ethical frameworks pertinent to good care of a diverse population.

\section{Acknowledgements}

I would like to express my gratitude to Professor Corrado Viafora and Dr. Silvia Tusino (Department of Molecular Medicine (DMM), University of Padova, who served as my supervisors. Special thanks go to Professor Pascal Borry, Phd, of the Interfaculty Centre for Biomedical Ethics and Law of the KU Leuven, who gave significant advice at all stages of this work. I acknowledge the European Commission, for the full Erasmus Mundus Scholarship, which enabled me to participate in the Erasmus mundus Bioethics course.

\section{Competing Interests Statement}

None to be declared.

\section{References}

Baldwin, C. (2015). Narrative ethics for narrative care. Journal of Aging Studies, 34, 183-189. https://doi.org/10.1016/j.jaging.2015.02.014

Bertolami, D. D. S. (2004). Why our ethics curricula don't work. Critical Issues in Dental Education, 68(4), 414-425.

Biazar, B. (2014). Critical/dialectical English as a Second Language education. Proceedings of the 2014 Annual Conference of Canadian Association for Study of Adult Education [CASAE/ACEEA], (Brock University, Ontario Canada, pp. 25-28).

Brookfield, S. (2002). Reassessing subjectivity, criticality and inclusivity: Marcuse's challenge to adult education. Adult Education Quarterly, 52, 265-280. https://doi.org/10.1177/074171302400448609

Brookfield, S. D., \& Preskill, S. (2005). Discussion as a way of teaching: Tools and techniques for democratic 
classrooms (2nd ed.). San Francisco, CA: Jossey-Bass.

Cannaerts, N., Gastmans, C., \& Dierckx de Casterle, B. (2014). Contribution of ethics education to the ethical competence of nursing students: Educators' and students' perceptions. Nursing Ethics, 21, 861-878. https://doi.org/10.1177/0969733014523166

Dirkx, J. M. (2006). Engaging emotions in adult learning: A Jungian perspective on emotion and transformative learning. New Directions in Adult Continuing Education, 109, 15-26. https://doi.org/10.1002/ace.204

Douglas, M. K., Pierce, J. U., Rosenkoetter, M., Pacquiao, D., Callister, L. C., Hattar-Pollara, M., \& Purnell, L. (2011). Standards of practice for culturally competent nursing care: 2011 update. Journal of Transcultural Nursing, 22(4), 317-333. https://doi.org/10.1177/1043659611412965

Esterhuizen, P. (2006). Is the professional code still the cornerstone of clinical nursing practice? Journal of Advanced Nursing, 53, 104-110. https://doi.org/10.1111/j.1365-2648.2006.03684.x

Geyer, N. (2015). A new approach to professional practice. Cape Town: Juta.

Kikuchi, J. F. (1996). Multicultural ethics in nursing education: A potential threat to responsible practice. Nursing Ethics, 12(3), 159-165. https://doi.org/10.1016/S8755-7223(96)80040-8

Kuhse, H., \& Singer, P. (2009). A companion to bioethics (2nd ed.). Oxford: Blackwell. https://doi.org/10.1002/9781444307818

Landis, K. (Ed.). (2008). Start talking. A handbook for engaging difficult dialogues in higher education (Anchorage: University of Alaska Anchorage, Alaska Pacific University).

Lawrence, R. L. (2008). Powerful feelings: Exploring the affective domain of informal and arts-based learning. New Directions for Adult \& Continuing Education, 120, 65-77. https://doi.org/10.1002/ace.317

Ludwick, R., \& Silva, M. C. (2000). Ethics: Nursing around the world: Cultural values and ethical conflicts. Online Journal of Issues in Nursing, 5(3). Retrieved from www.nursingworld.org/MainMenuCategories/ANAMarketplace/ANAPeriodicals/OJIN/Columns/Ethics/Cu lturalValuesandEthicalConflicts.aspx

Lutzen, K. (1997). Nursing ethics into the next millennium: A context-sensitive approach for nursing ethics. Nursing Ethics, 4(3), 218-226. https://doi.org/10.1177/096973309700400306

Mele, D., \& Sanchez-Runde, C. (2013). Cultural diversity and universal ethics in a global world. Journal of Business Ethics, 116, 681-687. https://doi.org/10.1007/s10551-013-1814-z

Merculieff, I., \& Roderick, L. (2013). Stop talking: Indigenous ways of teaching, learning, and difficult dialogues in higher education (Anchorage: The University of Alaska Anchorage).

Mezirow, J. (1997). Transformative learning: Theory to practice transformative learning theory. New Directions for Adult \& Continuing Education, 74, 5-12. https://doi.org/10.1002/ace.7401

Mezirow, J. (2003). Transformative learning as discourse. Journal of Transformative Education, 1, 58-63. https://doi.org/10.1177/1541344603252172

Owens, K. (2007). Classroom critiques: Transforming conformity into creativity. Industry and Higher Education, 21(5), 1-12. https://doi.org/10.5367/000000007782311786

Pattison, S., \& Wainwright, P. (2010). Is the 2008 NMC Code ethical? Journal of Nursing. Ethics, 17, 9-18. https://doi.org/10.1177/0969733009349991

Rachels, J., \& Rachels, S. (2012). The elements of moral philosophy (7th ed.). New York, NY: McGraw-Hill.

Squire, D. (2016). Engaging race and power in higher education organizations through a critical race institutional logics perspective. Journal of Critical Scholarship on Higher Education and Student Affairs, 2, 106-123.

Sue, D. W., Lin, A. I., Torino, G. C., Capodilupo, C. M., \& Rivera, D. P. (2009). Racial microaggressions and difficult dialogues on race in the classroom. Cultural Diversity and Ethnic Minority Psychology, 15(2), 183-190. https://doi.org/10.1037/a0014191

The University of Alaska Anchorage. Difficult Dialogues project: international initiative. 2012 and 2013. n.p. Retrieved from http://www.difficultdialoguesuaa.org/international_initiatives/init/south_africa

Walker, U. M. (1989). Moral understandings: Alternative 'epistemology' for a feminist ethics. Hypatia, 4, 15-28. https://doi.org/10.1111/j.1527-2001.1989.tb00570.x 
White, J., Phakoe, M., \& Rispel, L. C. (2015). "Practice what you preach": Nurses' perspectives on the code of ethics and service pledge in five South African hospitals. Global Health Action, 8, 1, 26341. https://doi.org/10.3402/gha.v8.26341

\section{Copyrights}

Copyright for this article is retained by the author(s), with first publication rights granted to the journal.

This is an open-access article distributed under the terms and conditions of the Creative Commons Attribution license (http://creativecommons.org/licenses/by/4.0/). 